\title{
A CHEOPS white dwarf transit search
}

\author{
Brett M. Morris ${ }^{1} \oplus$, Kevin Heng ${ }^{1}$, Alexis Brandeker ${ }^{2}$, Andrew Swan ${ }^{3}$, and Monika Lendl ${ }^{4}$ \\ ${ }^{1}$ Center for Space and Habitability, Gesellsschaftstrasse 6, 3012 Bern, Switzerland \\ e-mail: morrisbrettm@gmail.com \\ 2 Department of Astronomy, Stockholm University, AlbaNova University Center, 10691 Stockholm, Sweden \\ 3 Department of Physics and Astronomy, University College London, London WC1E 6BT, UK \\ 4 Observatoire de Genève, Université de Genève, Chemin des maillettes 51, 1290 Sauverny, Switzerland
}

Received 29 March 2021 / Accepted 12 May 2021

\begin{abstract}
White dwarf spectroscopy shows that nearly half of white dwarf atmospheres contain metals that must have been accreted from planetary material that survived the red giant phases of stellar evolution. We can use metal pollution in white dwarf atmospheres as flags, signalling recent accretion, in order to prioritize an efficient sample of white dwarfs to search for transiting material. We present a search for planetesimals orbiting six nearby white dwarfs with the CHaracterising ExOPlanet Satellite (CHEOPS). The targets are relatively faint for CHEOPS, $11 \mathrm{mag}<G<12.8 \mathrm{mag}$. We used aperture photometry data products from the CHEOPS mission as well as custom point-spread function photometry to search for periodic variations in flux due to transiting planetesimals. We detect no significant variations in flux that cannot be attributed to spacecraft systematics, despite reaching a photometric precision of $<2 \mathrm{ppt}$ in $60 \mathrm{~s}$ exposures on each target. We simulate observations to show that the small survey is sensitive primarily to Moon-sized transiting objects with periods between $3 \mathrm{~h}<P<10 \mathrm{~h}$, with radii of $R \gtrsim 1000 \mathrm{~km}$.
\end{abstract}

Key words. white dwarfs - instrumentation: photometers - techniques: photometric - planets and satellites: detection

\section{Introduction}

White dwarfs are the evolutionary end points of over $95 \%$ of all stars (Fontaine et al. 2001; Koester 2013). Given the ubiquity of small exoplanets orbiting Sun-like stars (Petigura et al. 2018), we seek to probe whether rocky planets survive stellar evolution and continue orbiting their host remnants into the white dwarf phase.

The existence of planetesimals and dust grains orbiting main sequence stars has been firmly established by the discovery and study of debris disks (for a review, see Wyatt 2008). Specifically, an infrared excess is detected relative to the stellar photosphere. The lifetimes of the dust grains associated with this infrared excess are typically orders of magnitude shorter than the disk lifetimes, implying the existence of (invisible) planetesimals that are constantly replenishing the dust population via collisions (Heng \& Tremaine 2010). An alternative interpretation of the infrared excess is that they are due to a large population of failed planetesimals with a sufficient covering fraction to produce an observable infrared excess (Heng \& Tremaine 2010). The study of debris disks motivates the study of observational signatures in white dwarfs that are of planetary origin.

There is clear spectroscopic evidence that rocky planetary material survives into the white dwarf phase (Zuckerman \& Reid 1998; Zuckerman et al. 2003, 2007; Farihi et al. 2008, 2009, 2010a,b, 2012; Hermes et al. 2014; Rocchetto et al. 2015). Spectroscopy reveals that nearly half of all white dwarfs show absorption lines due to metal pollution in their atmospheres (Wilson et al. 2019). Since metals have a finite lifetime in white dwarf atmospheres before sinking out of view, the presence of metals indicates recent accretion. Careful consideration of the chemical abundances present in white dwarf spectra have revealed that the accreted material must have been rock- and sometimes water-rich (Jura et al. 2009; Gänsicke et al. 2012; Farihi et al. 2013). Thus it appears that planetary bodies could survive into the white dwarf phase, which may provide a second era of habitability (Agol 2011).

Infrared excesses at some white dwarfs reveal the presence of debris disks containing $\sim 1000 \mathrm{~K}$ dust orbiting within $1 R_{\odot}$, which is much smaller and warmer than their main-sequence counterparts (Farihi 2016). These disks are found exclusively around polluted white dwarfs, and their infrared spectra have strong features from silicate grains, providing compelling evidence that the photospheric metals have a planetary origin (von Hippel et al. 2007; Jura et al. 2007; Farihi et al. 2012). The disks are hypothesized to be the end result of tidal disruptions of asteroids that have been perturbed onto highly eccentric orbits (Jura 2003).

Photometry of WD 1145+017 revealed disintegrating, likely rocky bodies orbiting a metal-polluted white dwarf (Vanderburg et al. 2015). Apparent transits of several dust clouds with orbital periods between 4 and $5 \mathrm{~h}$ were observed by the Kepler space telescope. The transit depths measured as large as $40 \%$, and varied from transit to transit (Izquierdo et al. 2018; $\mathrm{Xu}$ et al. 2018). This system demonstrates the great potential of metal-polluted white dwarf planetary systems: (1) the spectroscopic detection of the metal pollution enhances transit search efficiency by looking at only white dwarfs with recent accretion histories; (2) the geometrical advantage afforded by the small radii of white dwarfs produces deep transits from small objects; (3) the orbits where transiting material may be found have periods of only a few hours; and (4) each transit transmits unique 


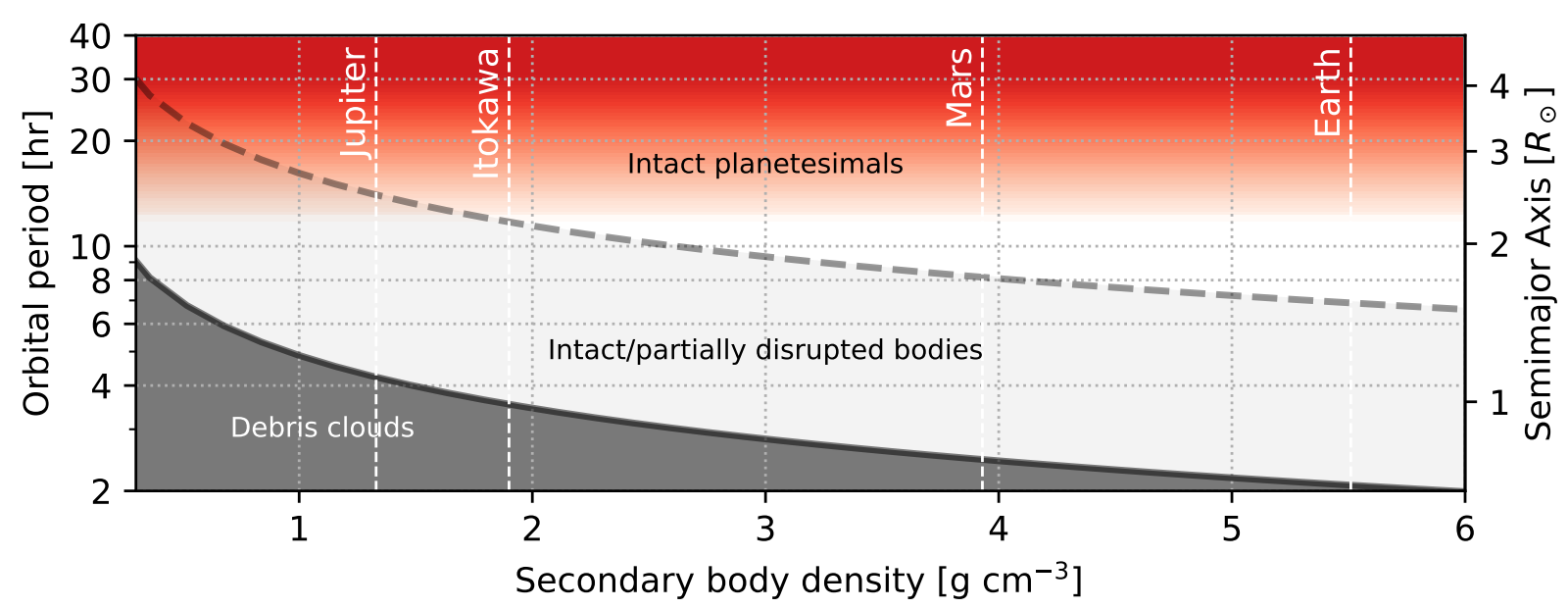

Fig. 1. Tidal disruption limits in period-density space for circular orbits (Veras et al. 2014). Vertical dashed lines mark the densities of several secondary bodies spanning a factor of five in bulk density from gas giant planets and rubble-pile asteroids through dense rocky bodies. Red regions of the plot represent regions where only single transit events may be detected in the CHEOPS photometry, nonred regions indicate where several occultations are possible within $24 \mathrm{~h}$ of photometry. The curves denote the regions where intact bodies may reside, the middle region marks potentially disrupted bodies, and black denotes bodies that we expect to be tidally disrupted.

information about the rapidly changing system, which appears to evolve from one orbit to the next. Since the 2015 discovery, several further detections of transiting material have been made including the discovery of 20 day-long eclipses with a period of 107 days in optical photometry of ZTF J013906.17+524536.89 (Vanderbosch et al. 2020), and the discovery of transits of WD $1856+534$ with a period of 1.4 days, corresponding to a Jupiter-sized occultor (Vanderburg et al. 2020).

The CHEOPS mission is a $30 \mathrm{~cm}$ effective-aperture telescope in low-Earth orbit since 2019 (Deline et al. 2020; Futyan et al. 2020; Hoyer et al. 2020). The spacecraft design is specified in Benz et al. (2021), and its initial performance is characterized by Lendl et al. (2020). In the first call for CHEOPS Guest Observer programs, we were awarded 84 orbits to search for transiting debris orbiting white dwarfs. We present the set of observations in AO-1 Program 7, completed in February 2021.

In Sect. 2, we provide a brief theoretical motivation for searches for transiting material on short orbital periods. In Sect. 3, we share two parallel analyses of the CHEOPS photometry with the data products produced by the CHEOPS team, as well as a reduction by a custom point-spread function (PSF) photometry package. In Sect. 4, we summarize a small simulation to estimate the detection efficiency of our CHEOPS observations in terms of sensitivity to planetsimal orbital period and radius. We discuss the implications of our observations in Sect. 5 and conclude in Sect. 6.

\section{Theoretical motivation}

The tidal disruption radius $r_{\mathrm{c}}$ for circular orbits is approximately

$\frac{r_{\mathrm{c}}}{R_{\odot}}=C\left(\frac{M_{\mathrm{WD}}}{0.6 M_{\odot}}\right)^{1 / 3}\left(\frac{\rho}{3 \mathrm{~g} \mathrm{~cm}^{-3}}\right)^{-1 / 3}$,

where $C$ is a constant ranging from about 0.85 to 1.89 (see e.g.: Veras et al. 2014; Bear \& Soker 2013). The period-density space of disrupted and intact bodies is shown in Fig. 1.

As we discuss later, we have observed the sample of white dwarfs in Table 1 for $24 \mathrm{~h}$ each, modulo the window function of Earth occultations. We required the minimum number of detected transits to be at least two in order to measure a periodic transiting event, so we only consider orbital periods $\lesssim 12 \mathrm{~h}$. With these observations in mind as a guide, Fig. 1 suggests that rocky planetesimals with bulk densities similar to most small asteroids, $\rho \approx 1.9 \mathrm{~g} \mathrm{~cm}^{-3}$ (Fujiwara et al. 2006), should be tidally disrupted on orbital periods of $P \lesssim 4 \mathrm{~h}$, may be tidally disrupted between $4 \mathrm{~h}<P<12 \mathrm{~h}$, and are likely intact otherwise.

By surveying white dwarfs for objects with orbital periods of $<12 \mathrm{~h}$, we are primarily sensitive to tidally disrupted material that has nearly circularized. This is consistent with the expectation that there is rocky material falling onto these white dwarfs based on the spectroscopic observations of metal pollution in their atmospheres.

\section{Observations}

The sample of metal-polluted white dwarfs observed with CHEOPS in AO-1 Program 7 is enumerated in Table 1. It includes six white dwarfs closer than $25 \mathrm{pc}$ which span a factor of four in $T_{\text {eff }}$.

In this work we consider the photometry produced with two methods, each processed with the same linear detrending as outlined in Sect. 3.1. In Sect. 3.2, we describe the standard aperture photometry products, and we revisit the same observations in Sect. 3.3 with PSF fitting photometry.

\subsection{Linear detrending}

The fluxes measured by the default aperture photometry from CHEOPS, $f$, contain several systematic trends as a function of the roll angle. To first order, the flux that we observe is a linear combination of the astrophysical signals we wish to detect and some unknown function of observational basis vectors. For some CHEOPS programs, the astrophysical signal has unconstrained quantities, such as transit times or depths. In principle, there is some design matrix $\mathbf{X}$, which we can solve for the least-squares estimators $\hat{\beta}$ such that

$\mathbf{X} \hat{\beta}=f$.

We provide a Python package to assemble a suitable design matrix from the CHEOPS data products called linea ${ }^{1}$, which by

1 https://github.com/bmorris3/linea 
Table 1. CHEOPS white dwarf survey sample and the light curve precision in one minute exposures.

\begin{tabular}{lccccrcrc}
\hline \hline WD & $\begin{array}{c}\text { Date } \\
{[U T C]}\end{array}$ & $\begin{array}{c}\text { Aperture } \\
\text { Radius [pix] }\end{array}$ & $\begin{array}{c}\text { Dist. } \\
\text { [pc] }\end{array}$ & $\begin{array}{c}\text { Gaia } G \\
\text { [mag] }\end{array}$ & $\begin{array}{r}T_{\text {eff }}{ }^{(1)} \\
{[\mathrm{K}]}\end{array}$ & $\begin{array}{c}\text { Spectral } \\
\text { Type }\end{array}$ & $\begin{array}{r}\text { DRP MAD } \\
\text { [ppt] }\end{array}$ & $\begin{array}{c}\text { PIPE MAD } \\
\text { [ppt] }\end{array}$ \\
\hline $0046+051$ & $2020-11-15$ & 25 & 4.3 & 12.31 & 6400 & DZ $^{(2)}$ & 1.65 & 1.25 \\
$1134+300$ & $2020-04-15$ & 33 & 15.7 & 12.51 & 22000 & DA $^{(3)}$ & 1.59 & 1.48 \\
$1202-232$ & $2021-02-15$ & 25 & 10.4 & 12.75 & 8700 & DAZ $^{(4)}$ & 1.98 & 0.98 \\
$1620-391$ & $2020-06-03$ & 25 & 12.9 & 11.00 & 26200 & DAZ $^{(5)}$ & 1.00 & 1.07 \\
$2032+248$ & $2020-07-04$ & 25 & 14.8 & 11.55 & 20400 & DAZ $^{(6)}$ & 1.03 & 1.66 \\
$2149+021$ & $2020-07-05$ & 25 & 22.5 & 12.79 & 18100 & DAZ $^{(7)}$ & 2.13 & 1.14 \\
\hline
\end{tabular}

References. ${ }^{(1)}$ Temperatures compiled by Gentile Fusillo et al. (2019), ${ }^{(2)}$ van Maanen (1917), ${ }^{(3)}$ McCleery et al. (2020), ${ }^{(4)}$ Zuckerman et al. (2003), ${ }^{(5)}$ Holberg et al. (1995), ${ }^{(6)}$ Wesemael et al. (1984), ${ }^{(7)}$ Koester et al. (2005).

default concatenates a design matrix of a unit vector, and the cosine and sine of the roll angle, and we added the product of the sine and cosine of the roll angle as an additional basis vector. The roll angle terms account for the variations in flux that occur as a result of the rotation of the spacecraft.

\subsection{CHEOPS data reduction pipeline $(D R P)$ photometry}

The DRP photometry contains corrections for dark and flat field normalization, bad pixels (pixels that are either hot or dead, and cosmic ray hits), and contamination from nearby stars in the aperture (Hoyer et al. 2020). After these corrections, the DRP measures the aperture photometry in four apertures of varying radii - we find that the DEFAULT aperture photometry produces a small median absolute deviation in the finished light curve, so we rely on that reduction for the DRP results presented here. The aperture radii for each target are listed in Table 1.

We masked outliers in the time series photometry by their corresponding stellar centroids by measuring the $4 \sigma$ interval over which most stellar centroids reside, and by rejecting any fluxes for which the centroid exceeds this radius from the mean centroid position. On average, we rejected $4 \%$ of fluxes with this approach.

The DRP photometry reduced with the linear detrending described in Sect. 3.1 is shown in the left panels of Fig. A.1, and the corresponding box-least squares (BLS) periodograms are shown in the panels on the right. The DRP photometry has typical median absolute deviations similar to 1 ppt in 1 min exposures. Periodic signals are detected in the photometry that can usually be ascribed to aliases of the CHEOPS orbital period of $100 \mathrm{~min}$ (marked with vertical dashed lines in the periodograms). We observed that periodic single-point outliers tend to occur at the beginning and end of an orbit, which correspond to the times when the Earth is closest to the CHEOPS field of view, when stray light can be expected to be greatest (see Appendix C).

\subsection{PSF photometry}

To get an independent photometric extraction and potentially improve the precision, we adopted the PSF extraction tool PSF Imagette Photometric Extraction (PIPE) to use on the subarrays. Some advantages of using PSF photometry compared to aperture photometry are the following: (1) it can properly weigh the contribution to the signal of each pixel within the aperture to reduce the impact of the readout noise; (2) it is easier to filter out the impact of hot pixels and cosmic rays by identifying outlier pixels and removing them from the fit; and (3) the background can be fit simultaneously with the PSF to be less sensitive to spatial variations. A potential problem with this technique is that it can introduce noise due to a mismatch between the model and the actual PSF. CHEOPS was built to have a stable PSF, but in particular jitter (typically $<1$ pix) will introduce motion blur during the $60 \mathrm{~s}$ exposures and modify the measured PSF. PIPE deals with motion blur by fitting a linear combination of model PSFs slightly offset from each other to the imagettes, improving the fit and leaving residuals dominated by photon noise. The model PSFs themselves were derived, one for each visit, by combining all observed frames within that visit.

The PIPE photometry, also reduced with the linear technique in Sect. 3.1, is shown in Fig. A.2. The PIPE photometry has typical median absolute deviations similar to $1 \mathrm{ppt}$ in one-minute exposures, similar to the DRP photometry, but it performs better by up to a factor of two for the fainter targets. As with the DRP photometry, periodic signals are detected in the photometry which are usually aliases of the CHEOPS orbital period (marked with vertical dashed lines).

\section{Transit simulations}

We determined the probability of detecting a single transiting object with time series photometry by generating a series of $10^{6}$ transit models, and by injecting them into the observations of our brightest and faintest targets using the open source Python package aspros ${ }^{2}$. We injected transits with the Mandel \& Agol (2002) transit light curve model with orbital periods ranging from $3 \mathrm{~h}<P<12 \mathrm{~h}$, transiting object radii between $500 \mathrm{~km}<$ $R<2000 \mathrm{~km}$, and assuming a fiducial white dwarf with $R_{\mathrm{WD}}=$ $9 \times 10^{8} \mathrm{~cm}$ and $M_{\mathrm{WD}}=0.6 M_{\odot}$.

We computed a BLS periodogram for each light curve, and measured the period with the strongest power and its aliases (Kovács et al. 2002). We measured the approximate signal-tonoise $(\mathrm{S} / \mathrm{N})$ of the candidate transit observations by measuring the significance of a transit model (fixed depth) compared with a transit-less model. If the $S / N>10$, we considered the transit detected.

The period distribution of detected transiting objects is shown in Fig. 2. Typical detection efficiency is near $90 \%$ at orbital periods shorter than 5 hours, though it drops to $40-60 \%$ when the orbital period of the transiting object is an integer multiple of the CHEOPS orbital period, and as the period approaches $12 \mathrm{~h}$.

The radius distribution of detected objects is shown in Fig. 3. The BLS technique is most sensitive to objects roughly $1000 \mathrm{~km}$ in size or larger, which is about $60 \%$ of the lunar radius. Objects

\footnotetext{
2 https://github.com/bmorris3/aspros
} 


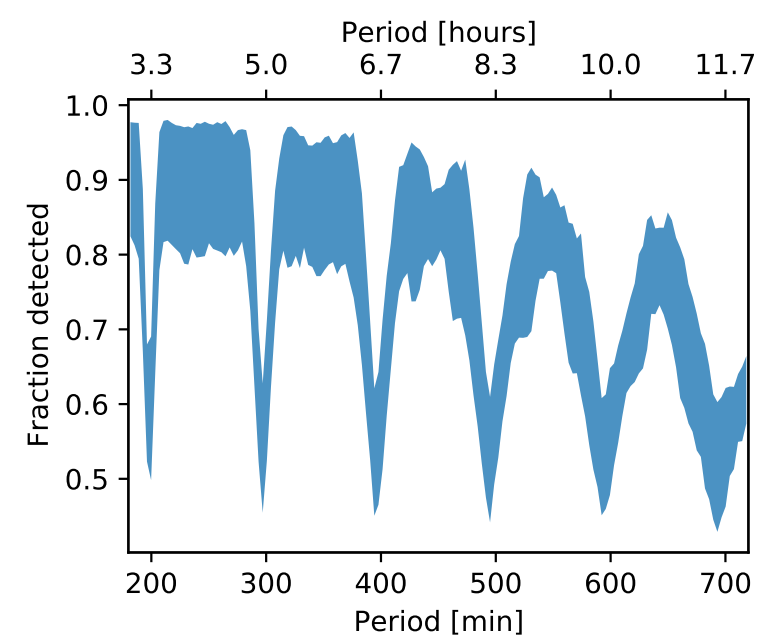

Fig. 2. Fraction of detected, simulated and injected transiting objects as a function of their orbital period. Aliases of the 100 min orbital period of CHEOPS are visible as drops in detection efficiency near integer multiples of $100 \mathrm{~min}$. The upper limit of the shaded region represents the fraction of objects detected for the brightest white dwarf in the sample, and the lower limit marks the detection fraction for the faintest white dwarf.

of this size can be thought of as monolithic planetesimals, rather than small rubble-piles asteroids (Lineweaver \& Norman 2010).

\section{Discussion}

\subsection{DRP versus PIPE photometry}

We have presented the results processed with the CHEOPS DRP and with the custom PSF photometry pipeline PIPE, see Figs. A.1 and A.2 and the median absolute deviations (MADs) in each light curve in Table 1 . In most cases, the DRP and PIPE photometry have similar MADs, though PIPE photometry yields smaller scatter than DRP photometry for the faintest targets by nearly a factor of two. We recommend PSF photometry for deep searches for transits with CHEOPS on faint targets $(V>12 \mathrm{mag})$, and for brighter targets the DRP photometry should suffice.

\subsection{Transit probability}

The geometric transit probability for an Earth-sized planet orbiting a $0.6 M_{\odot}$ white dwarf in a $250 \mathrm{~min}$ orbit is $3 \%$, implying that $\sim 30$ stars must be surveyed in order to have an appreciable chance of finding a single transiting object, assuming all white dwarfs host transiting material. As in Agol (2011), if we wanted to measure the white dwarf planet occurrence rate $\eta$ to a precision of 0.3 (e.g., nine planets discovered total), we would need to survey $\sim 300 \eta^{-1}$ white dwarfs. Even in the optimistic scenario that $\eta \approx 1$ for metal-polluted white dwarfs, we would require an observing program 50 times larger than the observations presented here to discover a new transiting planetesimal orbiting a white dwarf. For these reasons, we advocate here for continuing the transit search with wide-angle photometry from observatories such as TESS (Ricker et al. 2014), LSST (Lund et al. 2018), or Evryscope (Law et al. 2015), which combined will measure hundreds of light curves of white dwarfs. Narrow, deep campaigns such as the one presented here and in Wallach et al. (2018) for example, suggest that if transiting material were ubiquitous in orbit around white dwarfs, it would likely

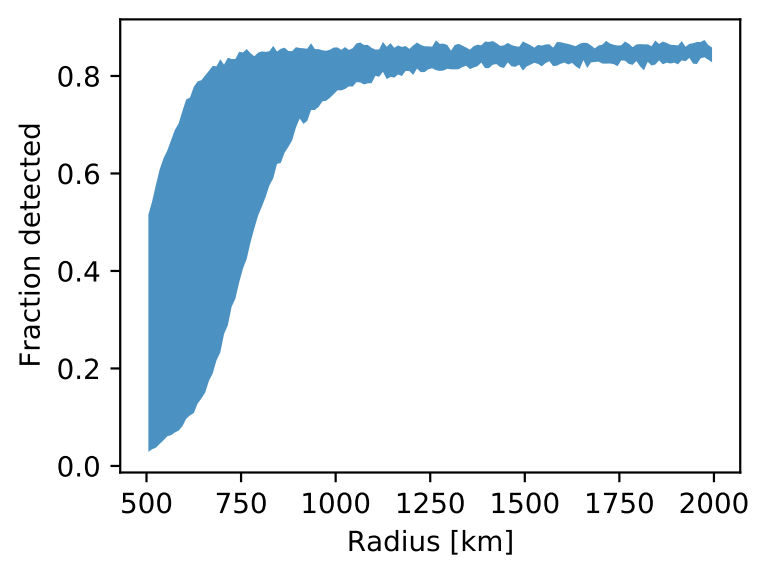

Fig. 3. Fraction of detected, simulated and injected transiting objects as a function of their radii. Objects with radii $>1000 \mathrm{~km}$ are more likely than not to be detected with $S / N>10$ around any of the six white dwarfs. As in Fig. 2, the upper and lower limits of the shaded region mark the detected fraction of transiting objects for the brightest and faintest of the six white dwarfs.

be quite small $\left(R_{p} \lesssim 1000 \mathrm{~km}\right)$ or orbiting at periods longer than about $P \gtrsim 8$ h.

\subsection{Dust-free systems}

By adapting the survival models of Heng \& Tremaine (2010), Veras \& Heng (2020) show that transit detections are possible in systems without significant dust disks by the delivery of intact planetesimals to close-in orbits at any cooling age. This reasoning suggests that transiting debris is perhaps likely orbiting white dwarfs regardless of metal pollution, so it may be fruitful to expand upon the work here to collect photometry of other bright, nearby white dwarfs that may host transiting debris without spectroscopic evidence of metal pollution.

\section{Conclusion}

We present the first search for transiting planetismals orbiting white dwarfs with the high precision photometry mission CHEOPS. The spacecraft can perform subpart-per-thousand photometry on targets as faint as $G=12.75$ mag with minimal data processing steps by the user. We find that our small sample of six metal-polluted white dwarfs showed no significant transitlike events in the $24 \mathrm{~h}$ that each target was observed. The exceptional quality photometry would have been sensitive to transiting material $\gtrsim 1000 \mathrm{~km}$ in radius on orbital periods $\lesssim 5 \mathrm{~h}$, but no such material was detected.

Acknowledgements. We are grateful for helpful mission support from Kate Isaak, and for valuable feedback from Jay Farihi. CHEOPS is an ESA mission in partnership with Switzerland with important contributions to the payload and the ground segment from Austria, Belgium, France, Germany, Hungary, Italy, Portugal, Spain, Sweden, and the United Kingdom. This work has been carried out in the framework of the PlanetS National Centre of Competence in Research (NCCR) supported by the Swiss National Science Foundation (SNSF). This research has made use of the VizieR catalogue access tool, CDS, Strasbourg, France (DOI: 10.26093/cds/vizier). The original description of the VizieR service was published in A\&AS 143, 23. AS acknowledges support from a Science and Technology Facilities Council studentship. We gratefully acknowledge the open source software which made this work possible: astropy (Astropy Collaboration 2013, 2018), ipython (Pérez \& Granger 2007), numpy (Harris et al. 2020), scipy (Virtanen et al. 2020), matplotlib (Hunter 2007), synphot (STScI development Team 2018), batman (Kreidberg 2015), lightkurve (Lightkurve Collaboration 2020). 
B. M. Morris et al.: A CHEOPS white dwarf transit search

\section{References}

Agol, E. 2011, ApJ, 731, L31

Astropy Collaboration (Robitaille, T. P., et al.) 2013, A\&A, 558, A33 Astropy Collaboration (Price-Whelan, A. M., et al.) 2018, AJ, 156, 123

Bear, E., \& Soker, N. 2013, New Astron., 19, 56

Benz, W., Broeg, C., Fortier, A., et al. 2021, Exp. Astron., 51, 109

Deline, A., Queloz, D., Chazelas, B., et al. 2020, A\&A, 635, A22

Farihi, J. 2016, New Astron. Rev., 71, 9

Farihi, J., Becklin, E. E., \& Zuckerman, B. 2008, ApJ, 681, 1470

Farihi, J., Jura, M., \& Zuckerman, B. 2009, ApJ, 694, 805

Farihi, J., Jura, M., Lee, J. E., \& Zuckerman, B. 2010a, ApJ, 714, 1386

Farihi, J., Barstow, M. A., Redfield, S., Dufour, P., \& Hambly, N. C. 2010b, MNRAS, 404, 2123

Farihi, J., Gänsicke, B. T., Wyatt, M. C., et al. 2012, MNRAS, 424, 464

Farihi, J., Gänsicke, B. T., \& Koester, D. 2013, Science, 342, 218

Fontaine, G., Brassard, P., \& Bergeron, P. 2001, PASP, 113, 409

Fujiwara, A., Kawaguchi, J., Yeomans, D. K., et al. 2006, Science, 312, 1330

Futyan, D., Fortier, A., Beck, M., et al. 2020, A\&A, 635, A23

Gänsicke, B. T., Koester, D., Farihi, J., et al. 2012, MNRAS, 424, 333

Gentile Fusillo, N. P., Tremblay, P.-E., Gänsicke, B. T., et al. 2019, MNRAS, 482,4570

Harris, C. R., Millman, K. J., van der Walt, S. J., et al. 2020, Nature, 585, 357

Heng, K., \& Tremaine, S. 2010, MNRAS, 401, 867

Hermes, J. J., Gänsicke, B. T., Koester, D., et al. 2014, MNRAS, 444, 1674

Holberg, J. B., Bruhweiler, F. C., \& Andersen, J. 1995, ApJ, 443, 753

Hoyer, S., Guterman, P., Demangeon, O., et al. 2020, A\&A, 635, A24

Hunter, J. D. 2007, Comput. Sci. Eng., 9, 90

Izquierdo, P., Rodríguez-Gil, P., Gänsicke, B. T., et al. 2018, MNRAS, 481, 703

Jura, M. 2003, ApJ, 584, L91

Jura, M., Farihi, J., \& Zuckerman, B. 2007, ApJ, 663, 1285

Jura, M., Muno, M. P., Farihi, J., \& Zuckerman, B. 2009, ApJ, 699, 1473

Koester, D. 2013, in White Dwarf Stars, eds. T. D. Oswalt, \& M. A. Barstow, 4, 559

Koester, D., Rollenhagen, K., Napiwotzki, R., et al. 2005, A\&A, 432, 1025

Kovács, G., Zucker, S., \& Mazeh, T. 2002, A\&A, 391, 369

Kreidberg, L. 2015, PASP, 127, 1161

Law, N. M., Fors, O., Ratzloff, J., et al. 2015, PASP, 127, 234
Lendl, M., Csizmadia, S., Deline, A., et al. 2020, A\&A, 643, A94

Lightkurve Collaboration (Cardoso, J. V. D. M., et al.), Lightkurve: Kepler and TESS time series analysis in Python

Lineweaver, C. H., \& Norman, M. 2010, ArXiv e-prints [arXiv:1004 . 1091]

Lund, M. B., Pepper, J. A., Shporer, A., \& Stassun, K. G. 2018, AAS J., submitted [arXiv:1809.10900]

Mandel, K., \& Agol, E. 2002, ApJ, 580, L171

McCleery, J., Tremblay, P.-E., Gentile Fusillo, N. P., et al. 2020, MNRAS, 499, 1890

Pérez, F., \& Granger, B. E. 2007, Comput. Sci. Eng., 9, 21

Petigura, E. A., Marcy, G. W., Winn, J. N., et al. 2018, AJ, 155, 89

Ricker, G. R., Winn, J. N., Vanderspek, R., et al. 2014, in Space Telescopes and Instrumentation 2014: Optical, Infrared, and Millimeter Wave, eds. J. Oschmann, M. Jacobus, M. Clampin, G. G. Fazio, \& H. A. MacEwen, SPIE Conf. Ser., 9143, 914320

Rocchetto, M., Farihi, J., Gänsicke, B. T., \& Bergfors, C. 2015, MNRAS, 449, 574

STScI development Team 2018, synphot: Synthetic photometry using Astropy, Astrophys. Source Code Libr. [record ascl:1811.001]

van Maanen, A. 1917, PASP, 29, 258

Vanderbosch, Z., Hermes, J. J., Dennihy, E., et al. 2020, ApJ, 897, 171

Vanderburg, A., Johnson, J. A., Rappaport, S., et al. 2015, Nature, 526, 546

Vanderburg, A., Rappaport, S. A., Xu, S., et al. 2020, Nature, 585, 363

Veras, D., \& Heng, K. 2020, MNRAS, 496, 2292

Veras, D., Leinhardt, Z. M., Bonsor, A., \& Gänsicke, B. T. 2014, MNRAS, 445, 2244

Virtanen, P., Gommers, R., Oliphant, T. E., et al. 2020, Nat. Meth., 17, 261

von Hippel, T., Kuchner, M. J., Kilic, M., Mullally, F., \& Reach, W. T. 2007, ApJ, 662, 544

Wallach, A., Morris, B. M., Branton, D., et al. 2018, Res. Notes. Am. Astron. Soc., 2,41

Wesemael, F., Henry, R. B. C., \& Shipman, H. L. 1984, ApJ, 287, 868

Wilson, T. G., Farihi, J., Gänsicke, B. T., \& Swan, A. 2019, MNRAS, 487, 133

Wyatt, M. C. 2008, ARA\&A, 46, 339

Xu, S., Rappaport, S., van Lieshout, R., et al. 2018, MNRAS, 474, 4795

Zuckerman, B., \& Reid, I. N. 1998, ApJ, 505, L143

Zuckerman, B., Koester, D., Reid, I. N., \& Hünsch, M. 2003, ApJ, 596, 477

Zuckerman, B., Koester, D., Melis, C., Hansen, B. M., \& Jura, M. 2007, ApJ, 671,872 


\section{Appendix A: Photometry and periodograms}

We present the photometry from the CHEOPS DRP and PIPE PSF photometry in Figs. A.1 and A.2.
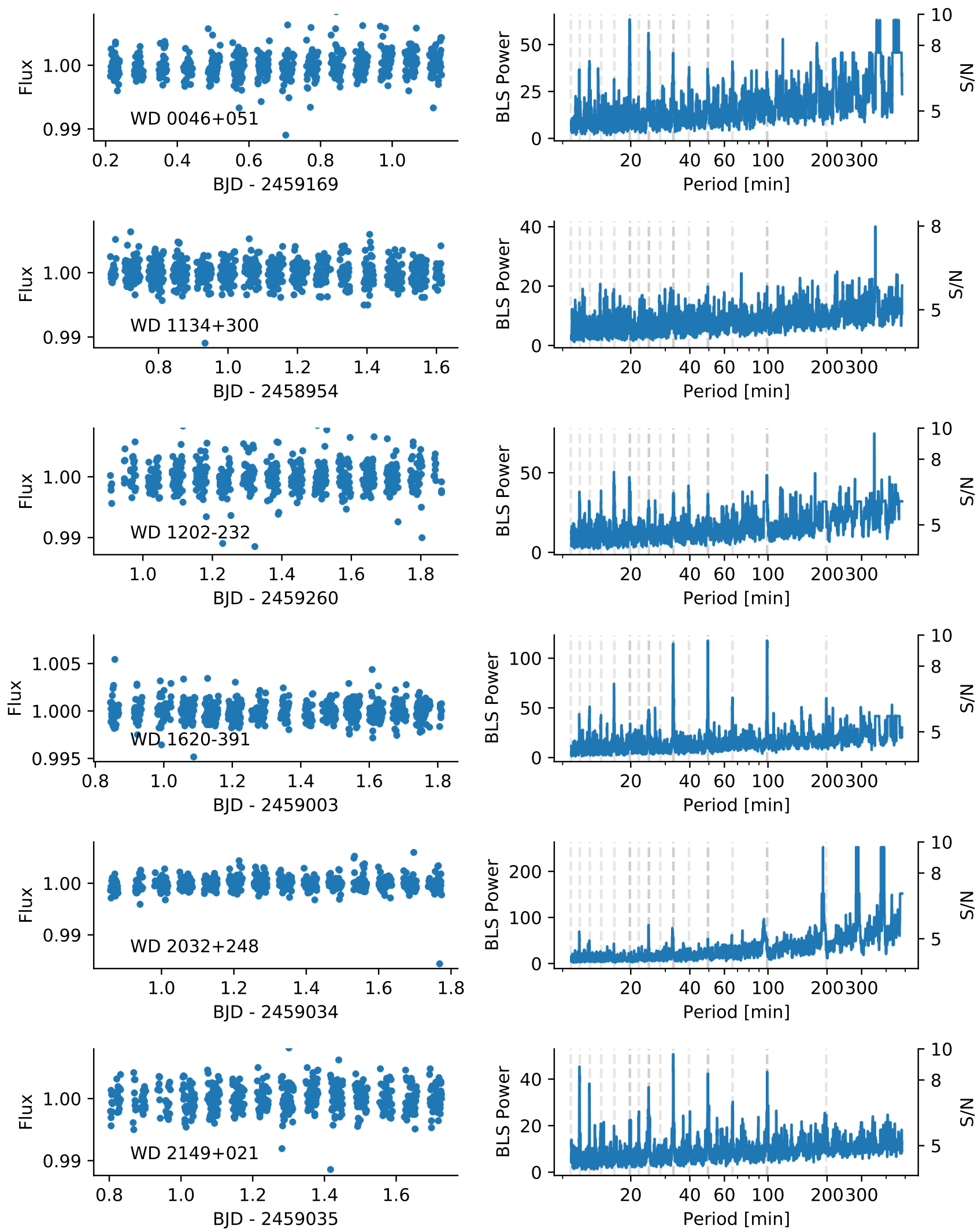

Fig. A.1. CHEOPS data reduction pipeline (DRP) light curves of six white dwarfs observed with the CHEOPS spacecraft in 2020 and 2021. Dashed vertical gray lines represent aliases of the CHEOPS orbital period ( 100 min). 
B. M. Morris et al.: A CHEOPS white dwarf transit search
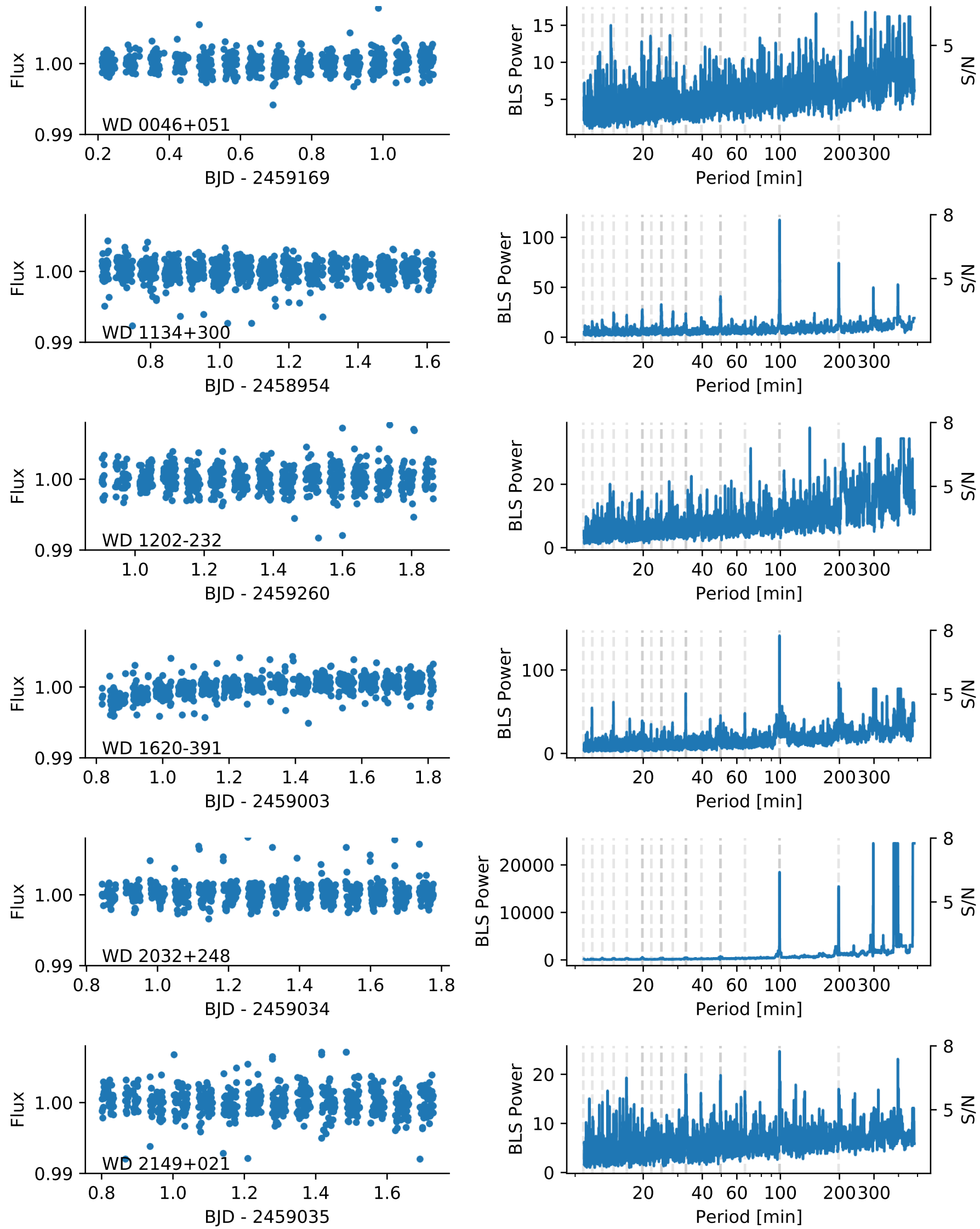

Fig. A.2. PIPE PSF photometry light curves of six white dwarfs observed with the CHEOPS spacecraft in 2020 and 2021. Compared with the DRP photometry in Fig. A.1, the PIPE photometry has a different sensitivity to detector artifacts and outliers. 


\section{Appendix B: Validation of the CHEOPS photometry} with TESS

Within about a year of each of the CHEOPS observations of these white dwarfs, the TESS spacecraft observed four out of the six white dwarfs studied in this work at two-minute cadence (Ricker et al. 2014). We performed a parallel analysis of these TESS observations to demonstrate that the CHEOPS non-detections presented above are in fact confirmed by the lack of photometric variability in the TESS observations.

We performed a similar BLS periodogram analysis to the one presented in Sect. 3 on the TESS observations and plot the results in Fig. B.1. No significant periodic events are detected with $S / N>5$, indicating that indeed there is no transiting material orbiting these white dwarfs on periods of 10-300 min.
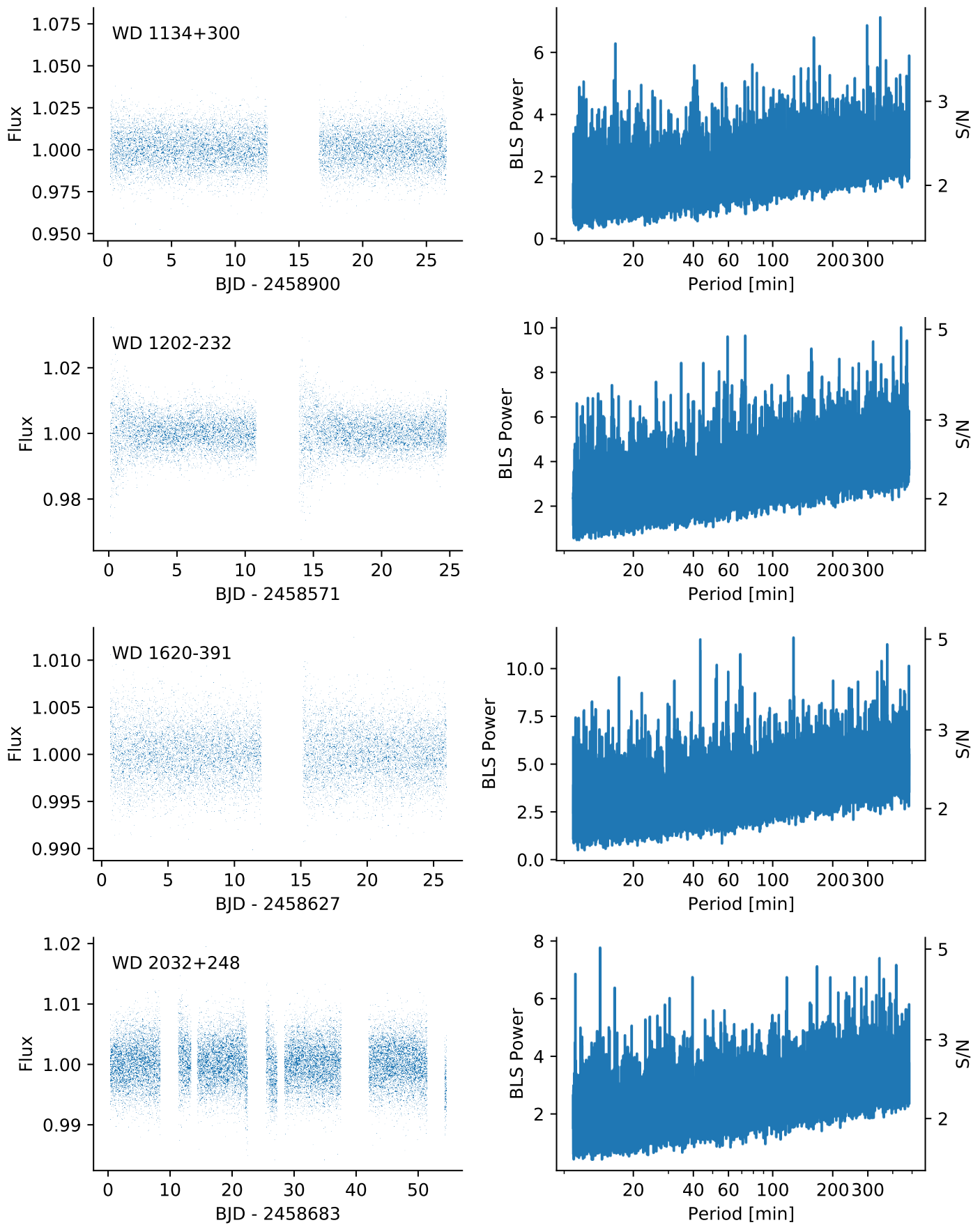

Fig. B.1. Same as Fig. A.1 presenting the two-minute cadence TESS observations of four of the six white dwarfs. No significant $S / N>5$ periodic events are detected, confirming the lack of variability reported with CHEOPS. 


\section{Appendix C: Masked fluxes}

Figure C. 1 shows the flux masking applied to the observations of WD $0046+051$. The most severe flux outliers occur when the stellar centroid is most distant from the mean centroid position and also at the roll angle corresponding to the time immediately before Earth occultation near the roll angle of $50^{\circ}$, where the expected scattered light is greatest.
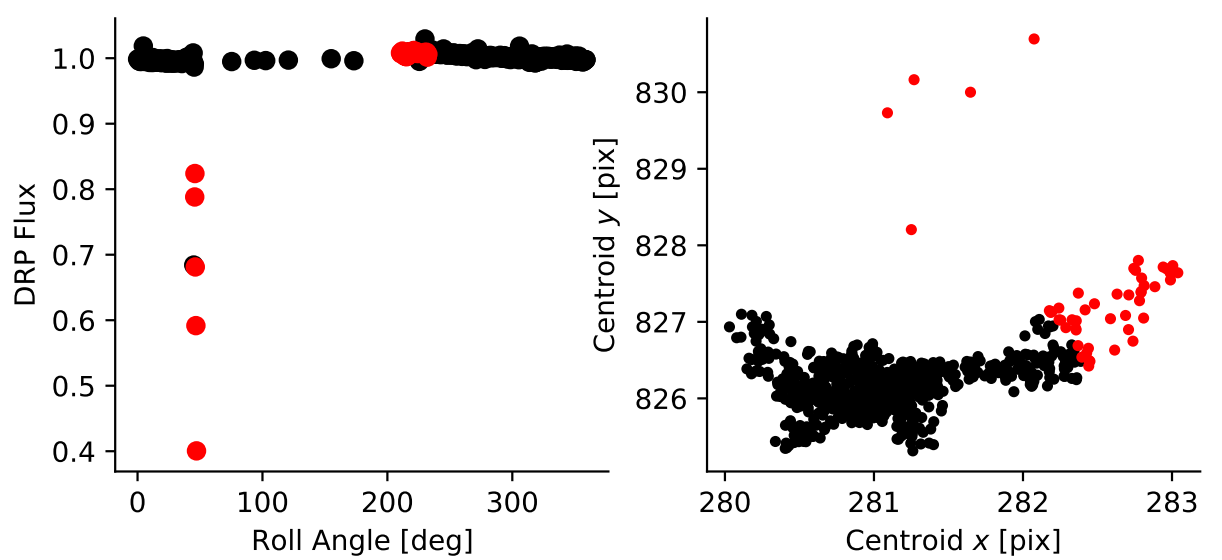

Fig. C.1. Masking of outlier fluxes (red) compared with typical observations (black) based on the $4 \sigma$ radius about the mean centroid. 